\title{
Upregulated epidermal growth factor receptor expression following near-infrared irradiation simulating solar radiation in a three-dimensional reconstructed human corneal epithelial tissue culture model
}

This article was published in the following Dove Press journal:

Clinical Interventions in Aging

I August 2016

Number of times this article has been viewed

\author{
Yohei Tanaka ${ }^{1,2}$ \\ Jun Nakayama ${ }^{2}$ \\ 'Department of Plastic Surgery, \\ Clinica Tanaka Plastic, Reconstructive \\ Surgery and Anti-aging Center, \\ ${ }^{2}$ Department of Molecular Pathology, \\ Shinshu University Graduate School \\ of Medicine, Matsumoto, Nagano, \\ Japan
}

Background and objective: Humans are increasingly exposed to near-infrared (NIR) radiation from both natural (eg, solar) and artificial (eg, electrical appliances) sources. Although the biological effects of sun and ultraviolet (UV) exposure have been extensively investigated, the biological effect of NIR radiation is still unclear. We previously reported that NIR as well as UV induces photoaging and standard UV-blocking materials, such as sunglasses, do not sufficiently block NIR. The objective of this study was to investigate changes in gene expression in three-dimensional reconstructed corneal epithelial tissue culture exposed to broad-spectrum NIR irradiation to simulate solar NIR radiation that reaches human tissues.

Materials and methods: DNA microarray and quantitative real-time polymerase chain reaction analysis were used to assess gene expression levels in a three-dimensional reconstructed corneal epithelial model composed of normal human corneal epithelial cells exposed to waterfiltered broad-spectrum NIR irradiation with a contact cooling $\left(20^{\circ} \mathrm{C}\right)$. The water-filter allowed 1,000-1,800 nm wavelengths and excluded 1,400-1,500 nm wavelengths.

Results: A DNA microarray with $>62,000$ different probes showed 25 and 150 genes that were up- or downregulated by at least fourfold and twofold, respectively, after NIR irradiation. In particular, epidermal growth factor receptor (EGFR) was upregulated by 19.4 -fold relative to control cells. Quantitative real-time polymerase chain reaction analysis revealed that two variants of EGFR in human corneal epithelial tissue were also significantly upregulated after five rounds of $10 \mathrm{~J} / \mathrm{cm}^{2}$ irradiation $(P<0.05)$.

Conclusion: We found that NIR irradiation induced the upregulated expression of EGFR in human corneal cells. Since over half of the solar energy reaching the Earth is in the NIR region, which cannot be adequately blocked by eyewear and thus can induce eye damage with intensive or long-term exposure, protection from both UV and NIR radiation may prevent changes in gene expression and in turn eye damage.

Keywords: DNA microarray, eye damage, gene expression, quantitative real-time PCR analysis, solar near-infrared

\section{Introduction}

Near-infrared (NIR) radiation can penetrate the skin and the sclera of the eye. The high permeability of NIR radiation also allows it to affect tissues deeper within the eye, such as muscles, the lens, and retina. NIR radiation can induce various biological 
effects, ${ }^{1-9}$ and intensive or long-term exposure to NIR radiation is a factor in premature aging. Despite the wide prevalence of a variety of ultraviolet (UV) blocking materials, such as sunblock, sunglasses, glasses, films, and umbrellas, that are useful in protecting our tissue against UV exposure, NIR cannot be blocked sufficiently. ${ }^{8}$ Consequently, in the absence of suitable protection, NIR radiation can induce various kinds of tissue damage and diseases, such as cataracts and photoaging. ${ }^{7,8}$

The human cornea plays a critical role in refracting light onto the retina and also protects the eye against external agents. Since the epithelial layer of the cornea provides the first line of defense against environmental insults, the structural integrity of this layer is a key component of corneal function. ${ }^{10}$ Although UV-induced corneal damage has been described in many previous studies, ${ }^{11-15}$ the effects induced by NIR radiation on the cornea have not been thoroughly investigated.

We hypothesized that NIR irradiation simulating solar NIR radiation that reaches human tissues can induce changes in gene expression. To test this hypothesis, a three-dimensional reconstructed human corneal epithelial model with multilayered, corneal epithelium-like structure was used to simulate the human eye, and we evaluated DNA microarray and real-time polymerase chain reaction (PCR) analysis results from normal human corneal epithelial cells exposed to water-filtered broad-spectrum NIR irradiation to simulate solar NIR radiation that reaches the eye.

\section{Materials and methods NIR irradiation}

NIR irradiation was performed with a broadband NIR source (Titan; Cutera, Brisbane, CA, USA). The NIR device emits an NIR spectrum between $1,100 \mathrm{~nm}$ and $1,800 \mathrm{~nm}$, with water filtering to remove wavelengths between $1,400 \mathrm{~nm}$ and 1,500 $\mathrm{nm}$, and simulates solar NIR radiation that reaches the skin of humans on the Earth's surface. To avoid thermal effects, the sapphire contact cooling tip was set to a fixed temperature of $20^{\circ} \mathrm{C}$. In our previous in vitro study, ten rounds at $10 \mathrm{~J} / \mathrm{cm}^{2}$ using continuous energy single irradiation pulses of 4.3 seconds achieved drastic reduction in cell count. Therefore, we performed five rounds of NIR irradiation at $10 \mathrm{~J} / \mathrm{cm}^{2}$.

\section{Corneal epithelial model}

The three-dimensional reconstructed human corneal epithelial model (LabCyte CORNEA-MODEL) prepared from enzymatically digested normal human corneal epithelial tissues was purchased from Japan Tissue Engineering Corporation, Aichi, Japan as an in vitro model of corneal tissue. ${ }^{16}$ Cells were cultured in media (Assay Medium; Japan Tissue Engineering Corporation), which was changed every 2 days until the cultures reached subconfluence. ${ }^{16}$ The subconfluent corneal cells were then subcultured with trypsin and seeded on a cell culture insert containing a microporous membrane with a $0.4 \mu \mathrm{m}$ pore size. Corneal cells were cultivated between the air and liquid interface to form a multilayered, corneal epithelium-like structure. All cells were grown at $37^{\circ} \mathrm{C}$ in a humidified incubator with $5 \% \mathrm{CO}_{2}$.

\section{RNA extraction}

Total RNA from the corneal epithelial cells was extracted in QIAzol reagent (Qiagen NV, Venlo, the Netherlands) and spin-column purified using a RNeasy mini spin column (Qiagen NV). The RNA was extracted from control and irradiated groups, which consisted of six whole individual three-dimensional reconstructed human corneal epithelial models. The quantity and quality of the RNA samples were then measured using UV absorbance (NanoDrop Technologies, Wilmington, DE, USA), and the quality was also assessed using an Agilent 2100 Bioanalyzer Series II (Agilent Technologies, Santa Clara, CA, USA).

\section{cRNA synthesis, labeling, and purification}

Cy3-labeled cRNA samples were synthesized using the Low Input Quick Amp Labeling Kit (Agilent Technologies) according to the manufacturer's instructions. For each time point, 50 ng of total RNA was used to generate first-strand cDNA. After the denaturation $\left(10\right.$ minutes at $\left.65^{\circ} \mathrm{C}\right)$ and cRNA synthesis $\left(2\right.$ hours at $40^{\circ} \mathrm{C}$ ) steps, the reactions were incubated at $70^{\circ} \mathrm{C}$ for 15 minutes to inactivate the AffinityScript enzyme (Agilent Technologies). For labeling reactions, cRNA samples were each mixed with $6 \mu \mathrm{L}$ of transcription master mix cocktail containing Cy3-CTP and incubated at $40^{\circ} \mathrm{C}$ for 2 hours. Purification was performed using the RNeasy mini spin columns. Labeled cRNA was quantified on a Nanodrop ND-1000 (NanoDrop Technologies), and quality was also checked by Agilent 2100 Bioanalyzer Series II.

\section{Microarray analysis}

cRNA fragmentation mixtures were first created using $600 \mathrm{ng}$ of experimental sample. These mixtures were incubated at $60^{\circ} \mathrm{C}$ for 30 minutes. Hybridization mixtures were prepared by adding $2 \times$ hybridization buffer and mixing by pipetting. Hybridization was carried out at $65^{\circ} \mathrm{C}$ for 17 hours. Washing 
Table I EGFR, TTLL5, and PQLC2 primers used for quantitative real-time PCR analysis

\begin{tabular}{lllll}
\hline Probe name & Gene name & Systematic name & Forward primer & Reverse primer \\
\hline A_33_P335I955 & EGFR & NM_201282 & CCGACAGCTATGAGATGGAG & ATTCCGTTACACACTTTGCG \\
A_24_P321752 & TTLL5 & NM_0I5072 & GGGTCCTACCTCGAGCATAA & GCAGTCATTCTGTCCTGGAA \\
A_33_P338849I & PQLC2 & NM_00I040I25 & TACCTGCTGCACCACCTG & TGGATGGAGATGATGGTGTC \\
\hline
\end{tabular}

Abbreviations: EGFR, epidermal growth factor receptor; PCR, polymerase chain reaction; PQLC2, PQ loop repeat containing 2; TTLL5, tubulin tyrosine ligase-like family member 5 .

was performed using Agilent's Stabilization and Drying Solution. Slides were scanned using the microarray scanner (Agilent Technologies), and images were processed by the Feature Extraction software (Agilent Technologies) with background correction. Data were imported into GeneSpring, and normalization was performed with a 75 percentile shift. After normalization and filtering of the raw data output files, log-fold changes in gene expression in the NIR-irradiated group compared with the control group were analyzed using the GeneSpring GX 11.0 software (Agilent Technologies) to determine the upregulated and downregulated genes, using a twofold change as the cutoff. Upregulated and downregulated genes were further clustered into functional gene groups using the ontology analysis function with the GeneSpring GX 11.0 software. Microarray data were deposited into the Minimum Information About a Microarray Experiment (MIAME)-compliant Gene Expression Omnibus (GEO) database (accession number: GSE76500, http://www.ncbi. nlm.nih.gov/geo/query/acc.cgi?acc=GSE76500).

\section{Quantitative real-time PCR analysis}

Quantitative real-time PCR analysis was achieved using the DNA Engine Opticon 2 System (Bio-Rad Laboratories Inc., Hercules, CA, USA). All six whole individual three-dimensional reconstructed human corneal epithelial tissue culture models were used for quantitative realtime PCR analysis. The primer sequences of epidermal growth factor receptor (EGFR), tubulin tyrosine ligase-like family member 5 (TTLL5), and PQ loop repeat containing 2 (PQLC2) are given in Table 1. Since PQLC was ubiqitously and highly expressed in all samples, PQLC2 was used for normalization. The variant-specific primer sequences for the four EGFR variants are given in Table 2. cDNA templates were prepared for each RNA sample by reverse transcription reaction using SuperScript III (Thermo Fisher Scientific, Waltham, MA, USA). Each quantitative PCR ( $25 \mu \mathrm{L})$ contained $1 \mu \mathrm{L}$ of cDNA template, $1.5 \mu \mathrm{L}$ of forward and reverse primer ( $10 \mu \mathrm{M}$ each), $7.5 \mu \mathrm{L}$ of $2 \times$ master mix, and $3.5 \mu \mathrm{L}$ of nuclease-free water. The thermal cycling conditions included one cycle at $95^{\circ} \mathrm{C}$ for 5 minutes and 40 cycles at $95^{\circ} \mathrm{C}$ for 10 seconds, $60^{\circ} \mathrm{C}$ for 30 seconds, and $68^{\circ} \mathrm{C}$ for 30 seconds.

\section{Statistical analysis}

Significance was evaluated using the Mann-Whitney $U$-test. All statistical data are presented as the mean \pm SEM, and a $P$-value of $<0.05$ was considered to be statistically significant.

\section{Results}

To elucidate changes in genetic expression induced by exposure to NIR irradiation that simulates solar NIR radiation that reaches human eye tissues, DNA microarray analysis was carried out using RNA isolated from control and NIRirradiated corneal epithelial cells. Using $\sim 6.3 \times 10^{4}$ probes, we identified 52 upregulated genes and 98 downregulated genes in the NIR-irradiated group compared with the control group. Gene ontology analysis of these 150 genes identified 41 upregulated genes that encode proteins with receptorbinding properties (Table 3). Quantitative real-time PCR analysis was then performed for the two genes showing the highest degree of upregulation in the DNA microarray analysis: EGFR and TTLL5. EGFR showed a high degree of upregulation (19.4-fold) relative to control cells, although statistically significant upregulation of EGFR and TTLL5 was not observed ( $P=0.5127$ and $P=0.8273$, respectively) (Figure 1) using the primer sequences listed in Table 1.

Table 2 Primers used for quantitative real-time PCR analysis to analyze EGFR variants

\begin{tabular}{lllll}
\hline Probe name & Gene name & Systematic name & Forward primer & Reverse primer \\
\hline A_33_P3351955 & EGFR variant I & NM_005228.3 & GGAAGAAGCTTGCTGGTAGC & TCTGGAAGACTTGTGGCTTG \\
& EGFR variant 2 & NM_201282.1 & TTCACTGTCTGACTTTAGTCTCC & GTGTCAGGACTTTATTTGAAGC \\
& EGFR variant 3 & NM_201283.1 & TTGAGCTGAATTATCACATGAA & GAACAGGAAATATGTCGAAAAG \\
& EGFR variant 4 & NM_201284.1 & CAGGAGTCATGGGAGAAAAC & GAATAACATGGCTTTGAGAC \\
\hline
\end{tabular}

Abbreviations: EGFR, epidermal growth factor receptor; PCR, polymerase chain reaction. 
Table 3 Upregulated genes in the NIR-irradiated group as compared with the control group

\begin{tabular}{|c|c|c|}
\hline Probe name & $\begin{array}{l}\text { Fold change (NIR irradiated } \\
\text { group/control) }\end{array}$ & Gene name \\
\hline A_33_P3351955 & 19.384924 & Epidermal growth factor receptor \\
\hline A_24_P321752 & 9.591753 & Tubulin tyrosine ligase-like family, member 5 \\
\hline A_2I_P00I4646 & 7.402783 & ARAPI antisense RNA 2 \\
\hline A_32_P2I383I & 7.007086 & Striatin interacting protein 2 \\
\hline A_33_P3254708 & 6.3440003 & Rho GTPase activating protein 40 \\
\hline A_33_P3424467 & 5.524943 & Consortin, connexin sorting protein \\
\hline A_33_P32I 4052 & 5.515191 & STXBP5 antisense RNA I \\
\hline A_32_P44700I & 5.1329985 & Chromosome 15 open reading frame 65 \\
\hline A_23_P96285 & 3.8647346 & Receptor accessory protein I \\
\hline A_23_PIII657 & 3.838098 & Sonic hedgehog \\
\hline A_23_P484I4 & 3.4167788 & Cyclin Al \\
\hline A_23_PI52420 & 3.3141088 & Gsel coiled-coil protein \\
\hline A_33_P3280779 & 3.268676 & Transmembrane protein 254 \\
\hline A_23_P2I 2258 & 3.1161842 & Kininogen I \\
\hline A_33_P3250055 & 3.0366344 & Mitogen-activated protein kinase 12 \\
\hline A_33_P3628409 & 3.0126626 & DKFZp $434 \mathrm{H} 1419$ \\
\hline A_24_P94054 & 3.012153 & Serine/threonine kinase 4 \\
\hline A_23_P373799 & 3.0048454 & CWC22 spliceosome-associated protein homolog (S. cerevisiae) \\
\hline A_33_P3239084 & 2.9155593 & Family with sequence similarity 86, member $A$ \\
\hline A_33_P3285565 & 2.882729 & Claudin 3 \\
\hline A_33_P3414I22 & 2.8212333 & Zinc finger protein 260 \\
\hline A_33_P3334448 & 2.5918674 & Small nucleolar RNA, H/ACA box 62 \\
\hline A_33_P3289996 & 2.5722313 & Ubiquitin specific peptidase 45 \\
\hline A_23_P78458 & 2.4986203 & Zinc finger protein 350 \\
\hline A_23_P209426 & 2.4799447 & Trafficking protein, kinesin binding 2 \\
\hline A_23_P20225 & 2.4031472 & Ribonucleotide reductase M2 B (TP53 inducible) \\
\hline A_23_P317056 & 2.3642921 & NADH dehydrogenase, subunit 6 (complex I) \\
\hline A_33_P3257678 & 2.3144212 & Histone cluster $2, \mathrm{H} 3 \mathrm{a}$ \\
\hline A_23_P250002 & 2.2984264 & HECT domain and ankyrin repeat containing E3 ubiquitin protein ligase I \\
\hline A_23_P34754I & 2.2951477 & Glutamate receptor, ionotropic, $N$-methyl-D-aspartate $3 \mathrm{~A}$ \\
\hline A_33_P3261408 & 2.2560165 & Transmembrane inner ear \\
\hline A_33_P3267230 & 2.217625 & $\mathrm{TBCI}$ domain family, member $3 \mathrm{G}$ \\
\hline A_23_P35995 & 2.197076 & CXADR-like membrane protein \\
\hline A_23_PI49545 & 2.1729438 & Histone cluster 2, $\mathrm{H} 2$ be \\
\hline A_19_P00320719 & 2.1425538 & Mdm4 p53 binding protein homolog (mouse) \\
\hline A_33_P3368049 & 2.1341257 & Chromosome I open reading frame 233 \\
\hline A_23_P406385 & 2.123014 & F-box and leucine-rich repeat protein 16 \\
\hline A_23_PI43713 & 2.0802233 & Apolipoprotein B mRNA editing enzyme, catalytic polypeptide-like 3G \\
\hline A_33_P3312194 & 2.0800095 & Tubulin folding cofactor B \\
\hline A_24_PIII2160 & $2.06 \mid 4858$ & Uroplakin 3B \\
\hline A 23 PI60828 & 2.0397763 & Chromosome I open reading frame I59 \\
\hline
\end{tabular}

Abbreviation: NIR, near-infrared.

Meanwhile, when the expression of EGFR variants was assessed in a DNA microarray analysis, expression increased by 1.0-fold and 1.3-fold for EGFR variants 1 and 3, respectively. Upon inclusion of variant-specific primer sequences for EGFR (Table 2), quantitative real-time PCR analysis revealed that statistically significant upregulation of variants 1 and 3 was observed $(P<0.05)$, whereas there was no statistically significant upregulation of variants 2 and $4(P=0.1266$ and $P=0.5127$, respectively) (Figure 2).

\section{Discussion}

To the best of our knowledge, this is the first study that examines changes in genetic expression in three-dimensional reconstructed corneal epithelial tissue after NIR irradiation to simulate solar NIR radiation that reaches human eye tissues.
The biological effects of sun and UV exposure have been extensively investigated. However, over half of the solar energy is in the NIR range, and most of the UV-blocking materials cannot block NIR radiation. Moreover, humans are continuously exposed to artificial NIR radiation from sources, such as electrical appliances. ${ }^{17,18}$ Despite the prevalence of NIR radiation, the need for protective measures against this type of radiation has received less attention than that of UV.

Because solar NIR radiation is filtered by atmospheric water, ${ }^{19,20}$ water-filtering in experimental settings is indispensable to provide an accurate simulation of solar NIR radiation that reaches human tissues. ${ }^{5}$ Cooling is also recommended when investigating the effects of NIR radiation, as NIR radiation can increase surface temperatures and induce thermal effects. ${ }^{5}$ Indeed, without water filter or contact cooling, 
EGFR

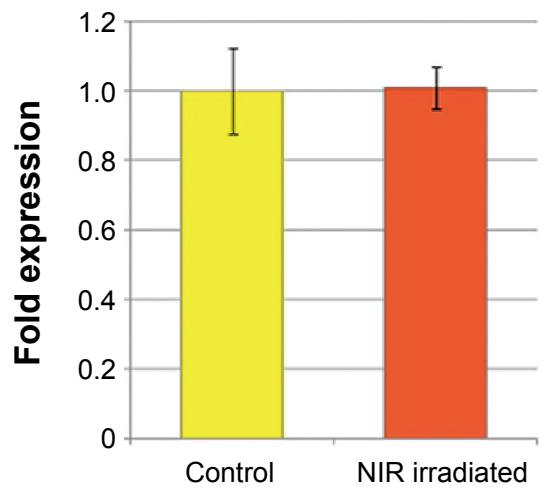

TTLL5

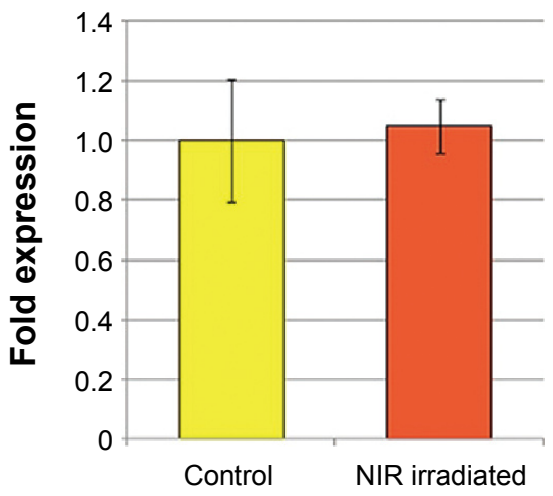

Figure I Quantitative real-time PCR validation of EGFR and TTLL5.

Notes: Fold-change in expression was calculated by setting the median value of expression seen in the control to I.0. Data are shown as the mean \pm SEM ( $n=3$ ).

Abbreviations: EGFR, epidermal growth factor receptor; NIR, near-infrared; PCR, polymerase chain reaction; SEM, standard error of the mean; TTLL5, tubulin tyrosine ligase-like family member 5 .

NIR irradiation immediately increases the temperature of the superficial layer of culture fluid in a laboratory dish or on skin, as NIR radiation is predominantly absorbed by hydrogen bond-containing molecules, such as water and hemoglobin. Meanwhile, as the NIR radiation penetrates deeper into the target, its energy can dissipate to the point where insufficient amounts of energy reach target cells in the base of culture dishes or deeper tissues in skin samples. Thus, to accurately investigate the biological effects of solar NIR radiation that reaches human tissues, a water-filter that excludes wavelengths between 1,400 $\mathrm{nm}$ and 1,500 $\mathrm{nm}$ and a cooling system were used in this study. ${ }^{5}$

A three-dimensional reconstructed human corneal epithelial model was used as an in vitro model of corneal tissue. The corneal epithelial model was proven to have a fully differentiated corneal epidermal tissue including all major corneal epithelial cell layers, such as the basal cell layer, the wing layer, and the superficial cell layer. Cytokeratin-3 is a
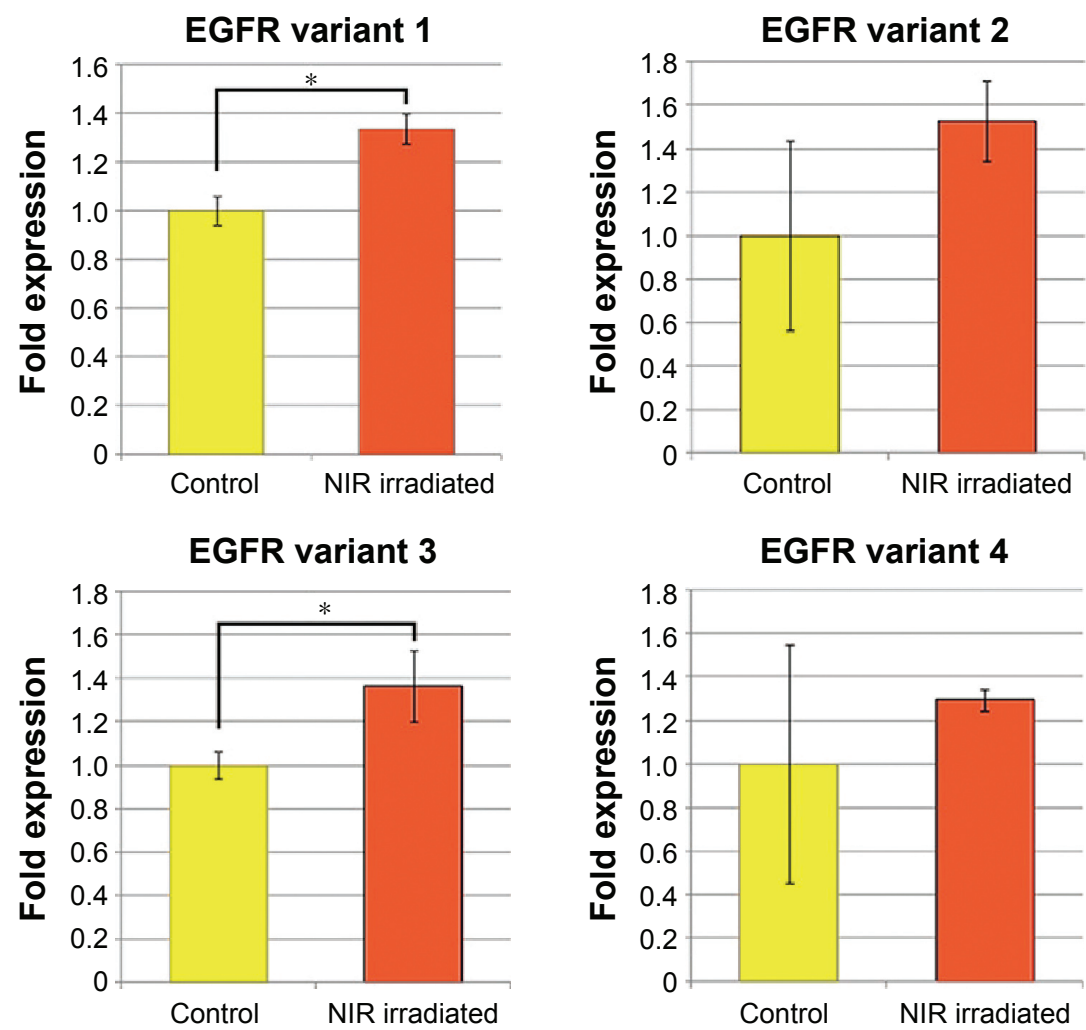

Figure 2 Quantitative real-time PCR validation of four EGFR variants.

Notes: Fold-change in expression was calculated by setting the median value of expression seen in the control to I.0. Data are shown as the mean $\pm S E M$ ( $n=3$ ). $* P<0.05$. Abbreviations: EGFR, epidermal growth factor receptor; NIR, near-infrared; PCR, polymerase chain reaction; SEM, standard error of the mean. 
specific marker of corneal epithelium, and it was expressed in all layers of the corneal epithelial model. ${ }^{16}$ MUC- 1 and MUC-16, which compose the transmembrane glycoproteins in the surface of the corneal epithelium, were well expressed in the superficial layer of the corneal epithelial model. ${ }^{16}$ Cells in the corneal epithelium are connected by desmosomes, tight junctions, and adherence junctions. ${ }^{16}$ Claudin-1, which is a marker of a tight junction, desmograin-3, which is a marker of desmosome, and E-cadherin, which is a marker of an adherence junction, are localized in the interface between cells at all cell layers, including the superficial layers. ${ }^{16}$ Lamin is an important constituent in the basement membrane at the basal corneal epithelium junction, which is expressed continuously in basal cells of the corneal epithelial model basal layer. ${ }^{16}$ The basement membrane was smooth like that of the human eye. ${ }^{16}$ Therefore, the corneal epithelial model reproduced many of the characteristics of the native human corneal tissue, and it provides a morphologically relevant means to assess NIR effects as an alternative to animal testing. The corneal epithelium, like other epithelial barriers in the human body, is continuously subjected to physical, chemical, and biological insults, which can produce a wound and/or loss of barrier functions. Proper healing of corneal wounds is vital for maintaining a clear, healthy cornea and preserving vision. Corneal epithelium responds rapidly to injury, wherein wound healing occurs by cells migrating as a sheet to cover the defect and reestablish barrier functions. ${ }^{21}$

In wounded cornea, the epithelium plays a central role, not only as a key cell type in corneal repair, but also as a growth factor source. ${ }^{22}$ As in other tissues, several growth factors are suggested to play a role in regulating corneal epithelial function and wound healing. ${ }^{21,23}$

Following NIR irradiation that simulates solar radiation, microarray analysis of corneal epithelial cell DNA in this study showed that EGFR was highly expressed, and statistically significant upregulation of EGFR variants 1 and 3 was observed $(P<0.05)$ by quantitative real-time PCR.

EGFR is a prototypic tyrosine kinase receptor that is part of a larger family of ErbB receptors. ErbB2, ErbB3, and ErbB4 share with EGFR characteristics that include extracellular ligand-binding sites, intracellular kinase domains, and tyrosine-rich regions. EGFR activated in response to injury serves as a powerful mediator of corneal epithelial wound healing ${ }^{10}$ by coordinating multiple extracellular signals generated in response to cell injury. ${ }^{24-27}$ In turn, the levels of EGFR and tyrosine kinase activity are major determinant factors for epithelial cell function in tissues and organs. ${ }^{22}$ Echoing this concept is the finding that in some cancer patients, treatment with EGFR-specific monoclonal antibodies cetuximab and gefitinib (an EGFR kinase inhibitor) resulted in ocular abnormalities, including diffuse punctate keratitis and corneal erosion. ${ }^{28-30}$ Thus, maintaining a proper level of EGFR signaling is critical for corneal homeostasis. ${ }^{22}$

TTLL5 gene has 32 exons with high expression in heart and skeletal muscle and lower expression in many other tissues, including the eye and brain..$^{31,32}$ It encodes a 1,281amino acid protein that is localized to the cytoplasm and nucleus. ${ }^{31}$ TTLL5 is thought to be a key initiator of polyglutamylation in $\alpha$-tubulin ${ }^{33}$ and has been previously reported to be essential for the correct function of sperm flagella. ${ }^{34}$ TTLL5 plays a role in polyglutamylation of primary cilia in vitro. ${ }^{35}$ Notably, genes involved in the polyglutamylation and deglutamylation of $\alpha$-tubulin have been associated with photoreceptor degeneration in mice. ${ }^{35}$

In this study, TTLL5 showed the high degree of upregulation in the DNA microarray analysis, whereas statistically significant upregulation of TTLL5 was not observed in quantitative real-time PCR analysis. Further studies are needed to determine if a higher power output or increased treatment frequency may promote significant upregulation of TTLL5. The findings here that EGFR expression is significantly upregulated following exposure of corneal epithelial cells to NIR irradiation support that solar NIR radiation that reaches human tissues can induce corneal cell injury. Although significant EGFR expression upregulation occurred after just one treatment at the tested power output, further studies are needed to determine if a higher power output or increased treatment frequency may promote even larger changes in expression. Furthermore, this was a preliminary study based on a fairly small number of samples, so a large-scale study will be needed.

\section{Conclusion}

NIR irradiation simulating solar NIR radiation that reaches human tissues induced upregulated expression of EGFR in human corneal cells. NIR radiation represents more than half of solar energy and cannot be sufficiently blocked by eyewear. Moreover, intensive or long-term NIR radiation exposure can induce photoaging. Thus, the results of this study indicate that enhanced protection from and avoidance of both UV and NIR radiation should be considered to prevent eye damage.

\section{Disclosure}

The authors report no conflicts of interest in this work. 


\section{References}

1. Tanaka Y, Matsuo K, Yuzuriha S, Shinohara H. Differential long-term stimulation of type I versus type III collagen after infrared irradiation. Dermatol Surg. 2009;35(7):1099-1104.

2. Tanaka Y, Matsuo K, Yuzuriha S. Long-term evaluation of collagen and elastin following infrared (1000 to $1800 \mathrm{~nm}$ ) irradiation. $J$ Drugs Dermatol. 2009;8(8):708-712.

3. Tanaka Y, Matsuo K, Yuzuriha S, Yan H, Nakayama J. Non-thermal cytocidal effect of infrared irradiation on cultured cancer cells using specialized device. Cancer Sci. 2010;101(6):1396-1402.

4. Tanaka Y, Tatewaki N, Nishida H, Eitsuka T, Ikekawa N, Nakayama J. Non-thermal DNA damage of cancer cells using near-infrared irradiation. Cancer Sci. 2012;103(8):1467-1473.

5. Tanaka Y. The impact of near-infrared radiation in dermatology. Review. World J Dermatol. 2012;1:30-37.

6. Tanaka Y, Tunemi Y, Kawashima M, Tatewaki N, Nishida H. Objective assessment of skin tightening using water-filtered near-infrared (1000-1800 nm) device with a contact cooling and freezer stored gel in Asians. Clin Cosmet Investig Dermatol. 2013;6:167-176.

7. Tanaka Y, Gale L. Beneficial applications and deleterious effects of near-infrared from biological and medical perspectives. Opt Photonics $J$. 2013;3:31-39.

8. Tanaka Y, Gale L. Protection from near-infrared to prevent skin damage. Opt Photonics J. 2015;5:113-118.

9. Tanaka Y, Motomura H, Jinno M. Biological defences against ultra-violet, visible light, and near-infrared exposure. Opt Photonics J. 2016;6: $8-14$.

10. Peterson JL, Phelps ED, Doll MA, Schaal S, Ceresa BP. The role of endogenous epidermal growth factor receptor ligands in mediating corneal epithelial homeostasis. Invest Ophthalmol Vis Sci. 2014; 55(5):2870-2880.

11. Cullen AP, Chou BR, Hall MG, Jany SE. Ultraviolet-B damages corneal endothelium. Am J Optom Physiol Opt. 1984;61(7):473-478.

12. Taylor HR. The biological effects of UV-B on the eye. Photochem Photobiol. 1989;50(4):489-492.

13. Wang F, Gao Q, Hu L, et al. Risk of eye damage from the wavelengthdependent biologically effective UVB spectrum irradiances. PLoS One. 2012;7(12):e52259.

14. Lennikov A, Kitaichi N, Kase S, et al. Induction of heat shock protein 70 ameliorates ultraviolet-induced photokeratitis in mice. Int J Mol Sci. 2013;14(1):2175-2189.

15. Hassan NM, Painter N, Howlett CR, et al. Brm inhibits the proliferative response of keratinocytes and corneal epithelial cells to ultraviolet radiation-induced damage. PLoS One. 2014;9(9):e107931.

16. Katoh M, Hamajima F, Ogasawara T, Hata K. Establishment of a new in vitro test method for evaluation of eye irritancy using a reconstructed human corneal epithelial model, LabCyte CORNEA-MODEL. Toxicol In Vitro. 2013;27(8):2184-2192.

17. Schieke SM, Schroeder P, Krutmann J. Cutaneous effects of infrared radiation: from clinical observations to molecular response mechanisms. Photodermatol Photoimmunol Photomed. 2003;19:228-234. Review article.

18. Schroeder P, Lademann J, Darvin ME, et al. Infrared radiation-induced matrix metalloproteinase in human skin: implications for protection. J Invest Dermatol. 2008;128(10):2491-2497.
19. Gates DM. Spectral distribution of solar radiation at the earth's surface. Science. 1966;151(3710):523-529.

20. Anderson RR, Parrish JA. The optics of human skin. J Invest Dermatol. 1981;77(1):13-19.

21. Lu L, Reinach PS, Kao WW. Corneal epithelial wound healing. Exp Biol Med. 2001;226(7):653-664.

22. Yu FS, Yin J, Xu K, Huang J. Growth factors and corneal epithelial wound healing. Brain Res Bull. 2010;81(2-3):229-235.

23. Imanishi J, Kamiyama K, Iguchi I, Kita M, Sotozono C, Kinoshita S. Growth factors: importance in wound healing and maintenance of transparency of the cornea. Prog Retin Eye Res. 2000;19(1):113-129.

24. Block ER, Klarlund JK. Wounding sheets of epithelial cells activates the epidermal growth factor receptor through distinct short- and longrange mechanisms. Mol Biol Cell. 2008;19(11):4909-4917.

25. Boucher I, Yang L, Mayo C, Klepeis V, Trinkaus-Randall V. Injury and nucleotides induce phosphorylation of epidermal growth factor receptor: MMP and HB-EGF dependent pathway. Exp Eye Res. 2007; 85(1):130-141.

26. Xu KP, Ding Y, Ling J, Dong Z, Yu FS. Wound-induced HB-EGF ectodomain shedding and EGFR activation in corneal epithelial cells. Invest Ophthalmol Vis Sci. 2004;45(3):813-820.

27. Yanai R, Yamada N, Inui M, Nishida T. Correlation of proliferative and anti-apoptotic effects of HGF, insulin, IGF-1, IGF-2, and EGF in SV40transformed human corneal epithelial cells. Exp Eye Res. 2006;83(1): $76-83$.

28. Foerster CG, Cursiefen C, Kruse FE. Persisting corneal erosion under cetuximab (Erbitux) treatment (epidermal growth factor receptor antibody). Cornea. 2008;27(5):612-614.

29. Shah NT, Kris MG, Pao W, et al. Practical management of patients with non-small-cell lung cancer treated with gefitinib. J Clin Oncol. 2005; 23(1):165-174.

30. Specenier P, Koppen C, Vermorken JB. Diffuse punctate keratitis in a patient treated with cetuximab as monotherapy. Ann Oncol. 2007;18(5): 961-962.

31. He Y, Simons SS Jr. STAMP, a novel predicted factor assisting TIF2 actions in glucocorticoid receptor-mediated induction and repression. Mol Cell Biol. 2007;27(4):1467-1485.

32. Bosch Grau M, Gonzalez Curto G, Rocha C, et al. Tubulin glycylases and glutamylases have distinct functions in stabilization and motility of ependymal cilia. J Cell Biol. 2013;202(3):441-451.

33. van Dijk J, Miro J, Strub JM, et al. Polyglutamylation is a posttranslational modification with a broad range of substrates. $J$ Biol Chem. 2008;283(7):3915-3922.

34. Lee GS, He Y, Dougherty EJ, et al. Disruption of Ttl15/stamp gene (tubulin tyrosine ligase-like protein 5/SRC-1 and TIF2-associated modulatory protein gene) in male mice causes sperm malformation and infertility. J Biol Chem. 2013;288(21):15167-15180.

35. Sergouniotis PI, Chakarova C, Murphy C, et al. Biallelic variants in TTLL5, encoding a tubulin glutamylase, cause retinal dystrophy. Am J Hum Genet. 2014;94(5):760-769.
Clinical Interventions in Aging

\section{Publish your work in this journal}

Clinical Interventions in Aging is an international, peer-reviewed journal focusing on evidence-based reports on the value or lack thereof of treatments intended to prevent or delay the onset of maladaptive correlates of aging in human beings. This journal is indexed on PubMed Central, MedLine,

\section{Dovepress}

CAS, Scopus and the Elsevier Bibliographic databases. The manuscript management system is completely online and includes a very quick and fair peer-review system, which is all easy to use. Visit http://www.dovepress. com/testimonials.php to read real quotes from published authors. 